\title{
Multiscale approach to characterize mechanical properties of tissue engineered skin
}

\author{
S. Tupin ${ }^{* 1}$, J. Molimard ${ }^{2}$, V. Cenizo ${ }^{3}$, T. Hoc ${ }^{1}$, B. Sohm ${ }^{3}$ and H. Zahouani ${ }^{* 1}$ \\ ${ }^{1}$ Laboratoire de Tribologie et Dynamique des Systèmes (LTDS, CNRS UMR5513), \\ Université de Lyon, Ecole Centrale de Lyon, ENISE, 69134 Ecully, France \\ 2 Ecole Nationale Supérieure des Mines, CIS-EMSE, INSERM UMR1059, SAINBIOSE, \\ 42023 Saint-Etienne, France \\ ${ }^{3}$ BASF Beauty Care Solutions France S.A.S, 69366 Lyon Cedex 07, France \\ "simon.tupin@ec-lyon.fr \\ ** hassan.zahouani@ec-lyon.fr
}

\section{Abstract and key terms}

Tissue engineered skin usually consist of a multi-layered visco-elastic material composed of a fibrillar matrix and cells. The complete mechanical characterization of these tissues has not yet been accomplished. The purpose of this study was to develop a multiscale approach to perform this characterization in order to link the development process of a cultured skin to the mechanical properties. As a proof-of-concept, tissue engineered skin samples were characterized at different stages of manufacturing (acellular matrix, reconstructed dermis and reconstructed skin) for two different aging models (using cells from an 18- and a 61-year-old man). To assess structural variations, bi-photonic confocal microscopy was used. To characterize mechanical properties at a macroscopic scale, a light-load micro-mechanical device that performs indentation and relaxation tests was designed. Finally, images of the internal network of the samples under stretching were acquired by combining confocal microscopy with a tensile device. Mechanical properties at microscopic scale were assessed. Results revealed that adding cells during manufacturing induced structural changes, which provided higher elastic modulus and viscosity. Moreover, senescence models exhibited lower elastic modulus and viscosity. This multiscale approach was efficient to characterize and compare skin equivalent samples and permitted the first experimental assessment of the Poisson's ratio for such tissues.

Keywords: Reconstructed skin; Bi-photonic confocal microscopy; Indentation; Digital image correlation; Opto-mechanical system; Poisson's ratio 


\section{Introduction}

Skin tissue engineering has become an important field of research for medical and cosmetic applications. Most reconstructed skin models are validated by histological and biochemical characterization. The main limitation of existing models is their mechanical properties. Few mechanical studies have been performed that analyze the mechanical behavior of skin equivalents.

In vitro skin models are usually dermis-epidermis composites, which are based on threedimensional scaffolds that are colonized with dermal cells (fibroblasts) and covered with epidermal cells (keratinocytes). The 3D scaffold consists of either a natural polymer, synthetic polymers, or a mixture of both polymer types and is assembled to mimic the fibrillar structure of the dermis, which supports and anchors dermal cells. ${ }^{5,6,16}$ Therefore, the 3D scaffold is used as a template to guide cell growth and tissue development. Several parameters can be modulated to influence tissue formation, such as the polymer and its chemical composition, porosity and pore interconnectivity, 3D organization and mechanical properties. ${ }^{14,21,23}$

Because of the complex structure of skin, the mechanical behavior is mainly attributed to the structure of the dermal layer. ${ }^{9}$ Indeed, the fibrillar network, which is composed of collagen and elastin fibers, is linked together by physiological cross-linking and contributes to the resistance and elasticity of the tissue. ${ }^{24,25}$ The surrounding medium, composed of proteoglycans and water, allows the fibers to slide against each other and therefore imparts viscous properties to the skin. The upper layer of the skin, the epidermis, has a stratified and cohesive structure resulting from the differentiation of keratinocytes. Engineered in vitro models have been used to demonstrate that the epidermal layer contributes to the mechanical properties of the entire skin when tensile tests are performed. ${ }^{8}$ Skin equivalent tissues present a visco-elastic mechanical behavior. Many techniques, inspired from material science, have been developed to characterize the behavior of soft biological tissues, such as stress relaxation, ${ }^{12}$ static indentation, ${ }^{19}$ dynamic indentation ${ }^{2}$ and oscillating shear tests. ${ }^{15}$ Moreover, to characterize the viscous behavior of such biological materials, relaxation tests are often used. ${ }^{11,20}$ The optical visualization of the micro-structure is essential to understand the complexity of these biological samples. Only a few experiments have been performed that combined optics and mechanical testing. $3,10,22,27$

In the present study, an original multiscale approach is developed to mechanically characterize tissue engineered skin and link its development process to the mechanical properties. Skin equivalent samples were characterized at different stages of manufacturing (acellular matrix, reconstructed dermis, reconstructed dermo-epidermis composite) for two different aging models (using cells from an 18- and a 61-year-old man). Fives samples of each type were produced. Bi-photonic confocal microscopy was firstly used to visualize the internal fibrillar and cellular network of the artificial samples. To characterize the global mechanical behavior of in vitro samples, a specific micro indentation/relaxation device was designed. Indentations were performed to characterize the elastic behavior of samples. The slope from the maximum of the unloading curve was used to determine the contact stiffness $\left(K_{\mathrm{Z}}=\mathrm{d} F / \mathrm{d} \delta\right)$ and reduced Young's modulus $\left(E^{*}\right)$ by following Hertz theory or the Johnson-Kendall-Roberts approach in the case of 
adhesion. Relaxation tests were performed on each sample. Experimental relaxation curves were analyzed using the generalized Maxwell model to characterize the instantaneous elastic modulus and the equivalent viscosity. Five indentation and five relaxation tests were performed on each sample (50 tests per skin equivalent type in total).

Finally, an opto-mechanical experimentation was developed by coupling bi-photonic confocal microscopy with a tensile device. It allowed us to acquire images of deformations in the micro-structure of the in vitro samples under unloaded and loaded (applied strain level of $2.5 \%$ ) conditions. Digital image correlation was used to obtain displacement and strain fields for this specific area. It allowed the experimental assessment of the Poisson's ratio. After combining these data with the mechanical properties obtained by indentation, we were able to assess the internal stress and force on the fibers during tensile testing.

\section{Materials and Methods}

\subsection{Material}

\subsubsection{Sample Types}

This study was performed using reconstructed skin samples licensed by BASF ${ }^{\circledR}$ Beauty Care Solutions, France. To evaluate the effect of sample structure on the mechanical properties, we mechanically characterized the acellular matrix, the reconstructed dermis and the reconstructed dermo-epidermis composite (reconstructed skin). Moreover, aging is a major concern in skin study. It is related to patient way of life, exposure to sun or pollution, and to his/her age. Here, in the context of reconstructed skin, it has been chosen to mimic the effect of aging by performing twice the entire process using cells from donors of different ages: an 18- and 61-year-old man. Fives samples of each type were produced.

\subsubsection{Sample Preparation}

The preparation of the reconstructed skin follows the work of Duplan-Perrat et al. ${ }^{7}$ and is summarized in Fig. 1.

Human keratinocytes and fibroblasts were isolated from human skin obtained from plastic surgery. Fibroblasts were grown in Dulbecco's modified Eagle's medium (DMEM with Glutamax-1, Life Technologies, Cergy-Pontoise, France) supplemented with 10\% calf serum (HyClone, Logan, USA) and $100 \mu \mathrm{g} / \mathrm{ml}$ antibiotics (Normocin, InvivoGen, San Diego, USA). Keratinocytes were grown to confluence in defined K-SFM medium (Life Technologies) and antibiotics.

Dermal equivalent fibroblasts were seeded at a density of 250,000 cells $/ \mathrm{cm}^{2}$ onto a dermal substrate consisting of a collagen-chitosan-glycosaminoglycan matrix (MIMEDISK $^{\mathrm{TM}}$ BASF BCS, France). This reconstructed dermis (MIMEDERM ${ }^{\mathrm{TM}}$ BASF BCS, France) was grown for 28 days at $37{ }^{\circ} \mathrm{C}$ in a $5 \% \mathrm{CO}_{2}$ atmosphere. The fibroblast DMEM medium (Life technologies) was supplemented with $10 \%$ fetal calf serum (FCS HyClone, Logan, USA), $10 \mathrm{ng} / \mathrm{ml}$ epidermal growth factor, $50 \mu \mathrm{g} / \mathrm{ml}$ L-ascorbic acid 
(Sigma, Saint Louis USA), and antibiotics (Invivogen). The medium was changed every day.

Keratinocytes were seeded on the dermal equivalent on day 28 at a density of 250000 cells $/ \mathrm{cm}^{2}$. Briefly, the cell-seeded matrices were submerged in keratinocyte medium consisting of a 3:1 mixture of DMEM and Ham's F12 (Invitrogen) supplemented with 10\% calf serum (HyClone), $10 \mathrm{ng} / \mathrm{ml}$ epidermal growth factor (Sigma), $0.12 \mathrm{IU} / \mathrm{ml}$ insulin (Lilly, Saint-Cloud, France), $0.4 \mu \mathrm{g} / \mathrm{ml}$ hydrocortisone, $2 \mathrm{ng} / \mathrm{ml}$ triiodo-thyronine, $5 \mu \mathrm{g} / \mathrm{ml}$ apotransferrin, $0.4 \mu \mathrm{g} / \mathrm{ml}$ isoprenaline, $24.3 \mu \mathrm{g} / \mathrm{ml}$ adenine (Sigma) and antibiotics for 7 days. Next, this reconstructed skin (MIMESKIN ${ }^{\text {TM }}$ BASF BCS, France) was elevated to the air-liquid interface and cultured for 14 more days in a simplified keratinocyte medium containing DMEM supplemented with $0.8 \%$ bovine serum albumin (Sigma), $0.12 \mathrm{IU} / \mathrm{ml}$ insulin, $0.4 \mu \mathrm{g} / \mathrm{ml}$ hydrocortisone, $50 \mu \mathrm{g} / \mathrm{ml} \mathrm{L}$-ascorbic acid and antibiotics. The medium was changed every 2 days.

\section{Figure 1: Preparation of the tissue engineered samples provided by BASF $^{\circledR}$ Beauty Care Solutions, France.}

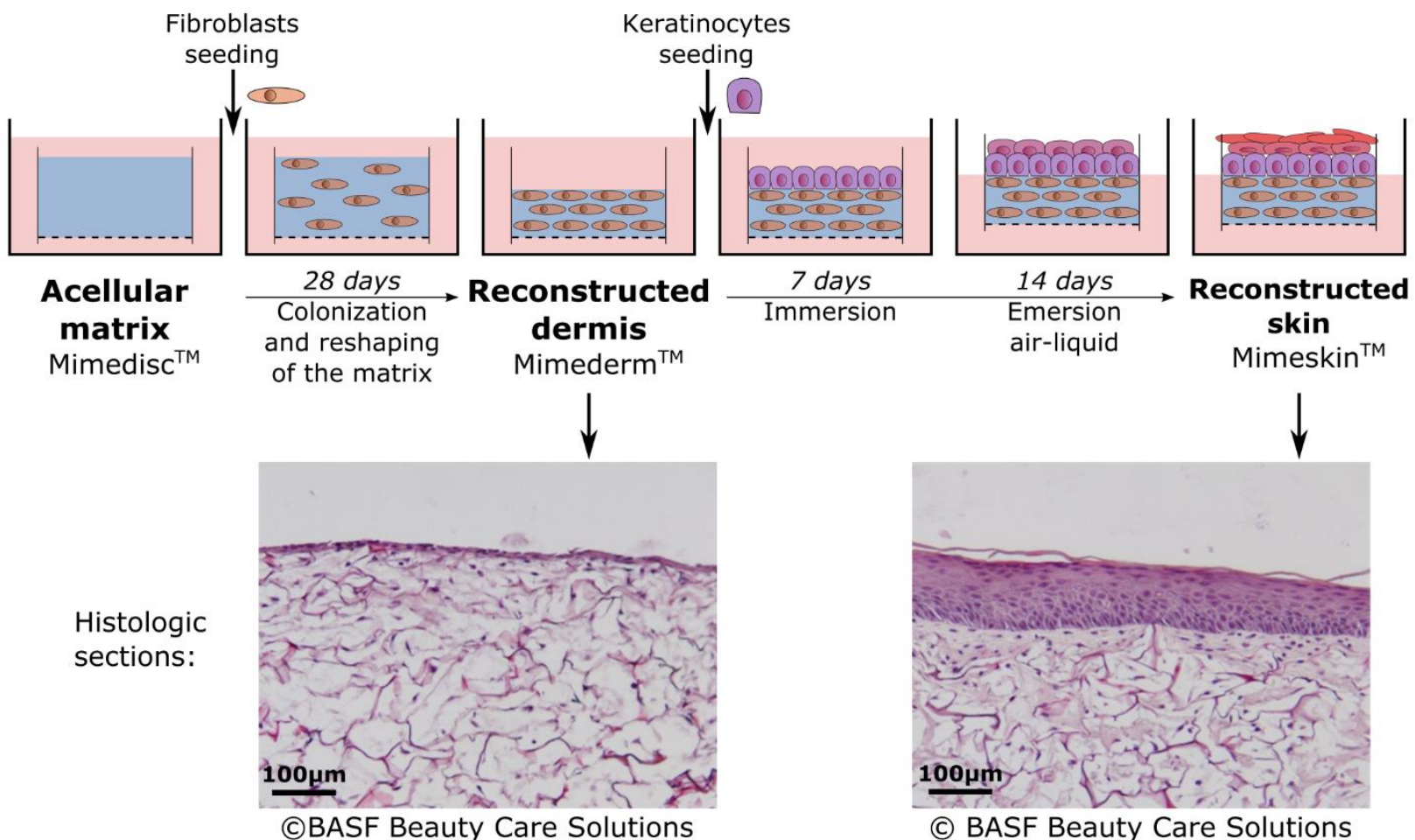

\subsubsection{Conservation Conditions}

Nine samples of each type were used in the preset work. All of the following tests were performed in an air-conditioned room with controlled temperature $\left(22-24^{\circ} \mathrm{C}\right)$ and relative humidity (30-40\%). Prior to testing, the samples were stored in an incubator at a constant temperature of $37^{\circ} \mathrm{C}$ and $5 \% \mathrm{CO}_{2}$. The tissue samples were removed from their culture 
plate and deposited on a glass slide for testing. Preliminary studies showed that mechanical properties are preserved at least 30 minutes; this time lapse is more than enough for the mechanical tests performed here.

\subsection{Confocal Microscopy}

A bi-photonic confocal microscope (Nikon, A1R MP Plus ${ }^{\circledR}$ ) was used to acquire 3D images of the internal network of collagen and cells in our samples. Two visualization channels with an excitation of $820 \mathrm{~nm}$ were used. The first channel (500 to $550 \mathrm{~nm}$ in blue) visualized the auto-fluorescence (AF) of the cells. A second channel (400 to $492 \mathrm{~nm}$ in green) allowed us to visualize collagen using second harmonic generation (SHG). Stack of images were captured with slice dimensions of $1024 \times 1024$ pixels at a resolution of $0.5 \mu \mathrm{m} / \mathrm{pixel}$ and $1 \mu \mathrm{m}$ between each slice. Density of signals were calculated for each $\mathrm{XY}$ slice as the percentage of pixels illuminated by each signal in the slice.

\subsection{Mechanical Experimentations}

\subsubsection{Device}

An original light-load indentation/relaxation device was developed for this type of test (previously described by Pailler-Mattei et al. ${ }^{20}$ ). The maximum force attained is $100 \mathrm{mN}$ with a resolution of $0.02 \mathrm{mN}$. This device offers a displacement with a resolution of $3 \mathrm{~nm}$ and indenting velocities from 1 to $100 \mu \mathrm{m} / \mathrm{s}$. A smooth PTFE spherical indenter with a radius of curvature $R=1.59 \mathrm{~mm}$ was selected as a probe to facilitate theoretical modeling and avoid adhesion between the probe and the biological sample.

\subsubsection{Assessment of the Indentation Curve and Determination of the Stiffness and Young's Modulus}

The principle of an indentation test is to push a rigid indenter perpendicularly into the sample in a load-unload displacement cycle at constant speed while the normal load, $F_{\mathrm{N}}$, vs. the displacement, $\delta$, was recorded (Fig. 2). Before each indentation test, the zero contact point of indentation was estimated by positioning the indenter tip immediately above the specimen surface and moving the tip downwards at a velocity of $5 \mu \mathrm{m} / \mathrm{s}$ until a change in load greater than $0.02 \mathrm{mN}$ was observed. The indenter was then raised by 1 $\mathrm{mm}$ to sever contact and moved down again to $200 \mu \mathrm{m}$ above the surface. Finally, the force was zeroed.

After the force was zeroed, a force-controlled indentation test was conducted with an indentation speed of $25 \mu \mathrm{m} / \mathrm{s}$ and a maximum load of $0.5 \mathrm{mN}$. This maximum load was chosen such that the classical semi-infinite assumption in indentation data extraction remained valid (indentation depth of less than $10 \%$ of the sample height). For each sample, five indentations were performed and the load-displacement $(\mathrm{N}-\mathrm{m})$ data were recorded at a sampling of $1 \mathrm{kHz}$ using specific software (based on LabVIEW ${ }^{\circledR}$ ).

Following Zahouani et al. ${ }^{29}$, the contact stiffness, $K_{\mathrm{Z}}[\mathrm{N} / \mathrm{m}]$ is the slope of the initial portion of the unloading curve (Fig. 2) that is determined by: 


$$
K_{\mathrm{Z}}=\left|\frac{\mathrm{d} F}{\mathrm{~d} \delta}\right|_{F_{\max }}
$$

where $F[\mathrm{~N}]$ is the normal load and $\delta[\mathrm{m}]$ is the displacement of the indenter. Following the Hertz theory, an approximation of the contact radius, $a[\mathrm{~m}]$, depending on the maximum displacement of the indenter, $\delta[\mathrm{m}]$, and the indenter radius, $R[\mathrm{~m}]$, is determined using Eq. (2):

$$
a=\sqrt{R \delta}
$$

Alternatively, the contact radius can by determined from the maximum load, $F_{\max }[\mathrm{N}]$, contact stiffness, $K_{\mathrm{Z}}[\mathrm{N} / \mathrm{m}]$, and indenter radius, $R[\mathrm{~m}]$, as follows:

$$
a=\sqrt{\frac{3 R F_{\max }}{2 K_{\mathrm{Z}}}}
$$

Then, we can determine the reduced Young's modulus, $E^{*}[\mathrm{~Pa}]$, using Eq. (4):

$$
E^{*}=\frac{K_{\mathrm{Z}}}{2 a}
$$

Note: The reduced Young's modulus is equal to:

$$
\frac{1}{E^{*}}=\frac{1-v_{1}^{2}}{E_{1}}+\frac{1-v_{2}^{2}}{E_{2}}
$$

where $E_{1}$ and $E_{2}$ are the Young's modulus of the sample and the Young's modulus of the spherical indenter, respectively, and $v_{1}$ and $v_{2}$ are the Poisson's ratio of the sample and the Poisson's ratio of the indenter, respectively. Therefore, $E_{2} » E_{1}$ and the reduced Young's modulus is: $\frac{1}{E^{*}}=\frac{1-v_{1}^{2}}{E_{1}}$.

When the probe adhered to the sample, a negative force was observed (Fig. 2) and the slope of the unloading curve changed. Johnson et al. ${ }^{13}$ developed a successful elastic model, designated as the JKR model, which helped to correct for this adhesive behavior during estimation of the elastic modulus.

The new approximation of the contact area, $a_{\mathrm{JKR}}[\mathrm{m}]$, is:

$$
a_{\mathrm{JKR}}=\sqrt[3]{\frac{3 R}{4 E^{*}}\left(F_{\max }+3 \pi R \Delta \gamma+\sqrt{6 \pi R \Delta \gamma F_{\max }+(3 \pi R \Delta \gamma)^{2}}\right)}
$$

where $\Delta \gamma$ is the work of adhesion.

The maximal adhesion load can be expressed as:

$$
F_{\mathrm{adh}}=-\frac{3}{2} \pi R \Delta \gamma
$$

Finally, the reduced elastic modulus can be obtained using Eq. (4), Eq. (6) and Eq. (7):

$$
E_{\mathrm{JKR}}^{*}=\sqrt{\frac{K_{\mathrm{Z}}^{3}}{6 R\left(F_{\max }-2 F_{\mathrm{adh}}-2 \sqrt{F_{\mathrm{adh}}\left(F_{\max }-F_{\mathrm{adh}}\right)}\right)}}
$$


Figure 2: Experimental indentation curve of a sample presenting a visco-elastic adhesive behavior and schematic of the indentor penetrating the sample.

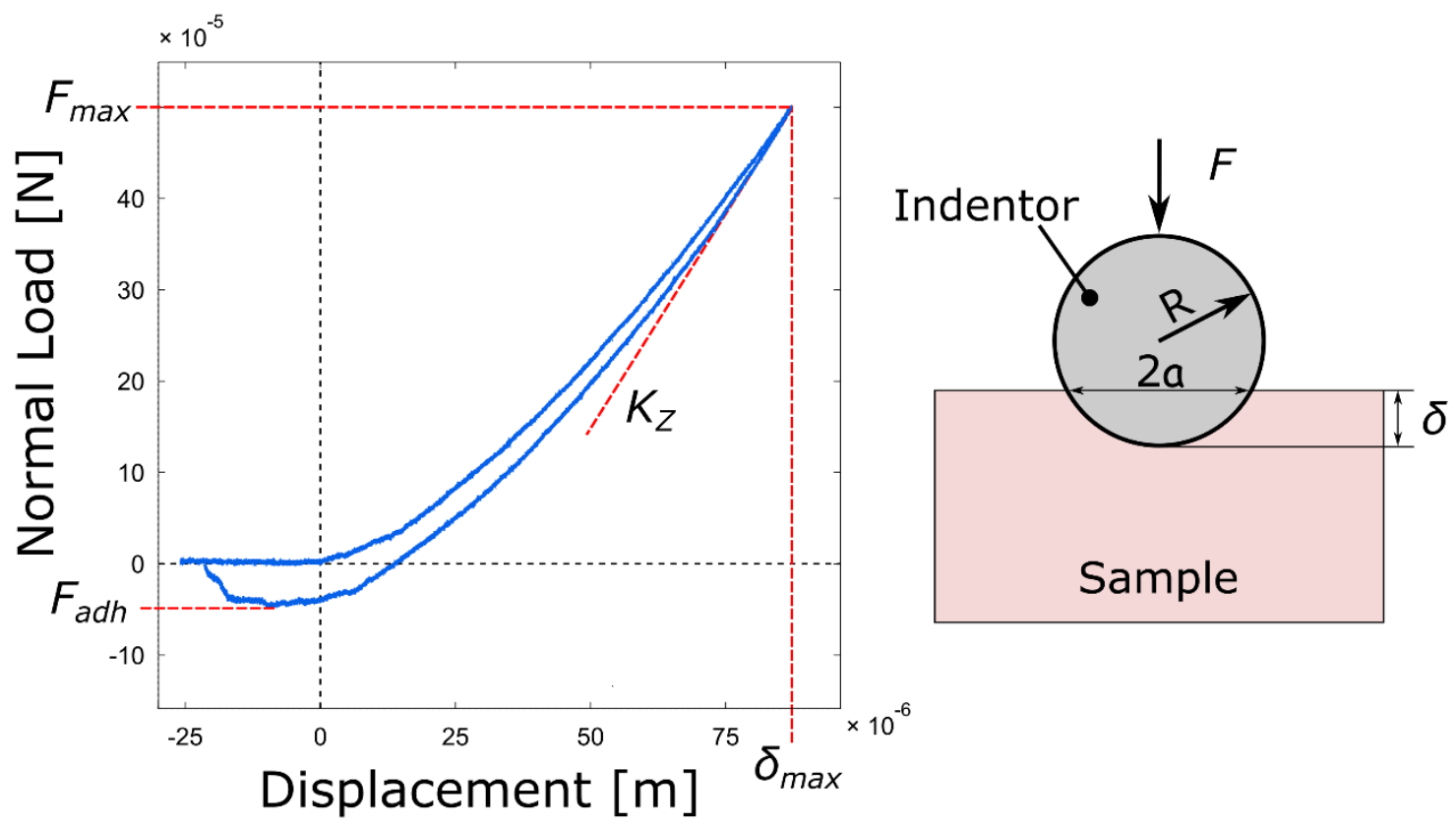

\subsubsection{Assessment of the Relaxation Curve and Determination of the Instantaneous Young's Modulus and Equivalent Viscosity}

A relaxation test is similar to an indentation test with a lag time between the loading and unloading parts of the curve. During this time, we followed the decrease in the normal load, which is the relaxation curve (Fig. 3).

We followed an identical pre-test procedure for indentation using the following parameters: indentation speed of $50 \mu \mathrm{m} / \mathrm{s}$, maximum load of $0.5 \mathrm{mN}$ and latency time of $100 \mathrm{~s}$. For each sample, five relaxation tests were conducted and load-time (N-s) data were recorded at a sampling of $1 \mathrm{kHz}$ using developed software (on LabVIEW ${ }^{\circledR}$ ).

The generalized Maxwell rheological model with two branches was applied to fit the relaxation part of the curves. Thanks to this model, the instantaneous elastic modulus, $E_{\text {ins }}[\mathrm{Pa}]$, and the equivalent viscosity, $\eta_{\text {equ }}[\mathrm{Pa}$.s], can be calculated according to the contact area.

The generalized Maxwell model consists of a spring that is connected in parallel to $n$ branches that contain a spring and a dashpot connected in series (Fig. 3). Based on the generalized Maxwell model, an analytical solution using a functional equation for the spherical indentation load-relaxation test was proposed by Pailler-Mattei et al. ${ }^{20}$ :

$$
F(\mathrm{t})=\frac{16}{3} R^{1 / 2} \delta_{0}{ }^{3 / 2}\left(G_{0}+\sum_{i=1}^{2} G_{i} \mathrm{e}^{-t / \tau_{i}}\right)
$$


where $\tau_{i}=\eta_{i} / G_{i}$. The experimental data obtained from the relaxation curves were fitted to the analytical models (Fig. 3) using the simplex method ${ }^{18}$ to obtain $G_{i}$ and $\eta_{i}$. The Young's moduli, $E_{i}$, can be deduced directly from $G_{i}$ :

$$
E_{i}=2 G_{i}\left(1+v_{1}\right)
$$

Finally, the instantaneous Young's modulus, $E_{\text {ins }}$, and equivalent viscosity, $\eta_{\text {equ }}$, can be obtained by reducing the model to a single branch:

$$
\begin{aligned}
& E_{\mathrm{ins}}=E_{0}+\sum_{i=1}^{2} E_{i} \\
& \eta_{\mathrm{eq}}=\frac{\left(\sum_{i=1}^{n} E_{i}\right)^{2}}{\sum_{i=1}^{n} E_{i} / \tau_{i}}
\end{aligned}
$$

Figure 3: Experimental relaxation curve of a sample presenting a visco-elastic behavior (in blue) and fitting curve using the generalized Maxwell model with two branches (in red).

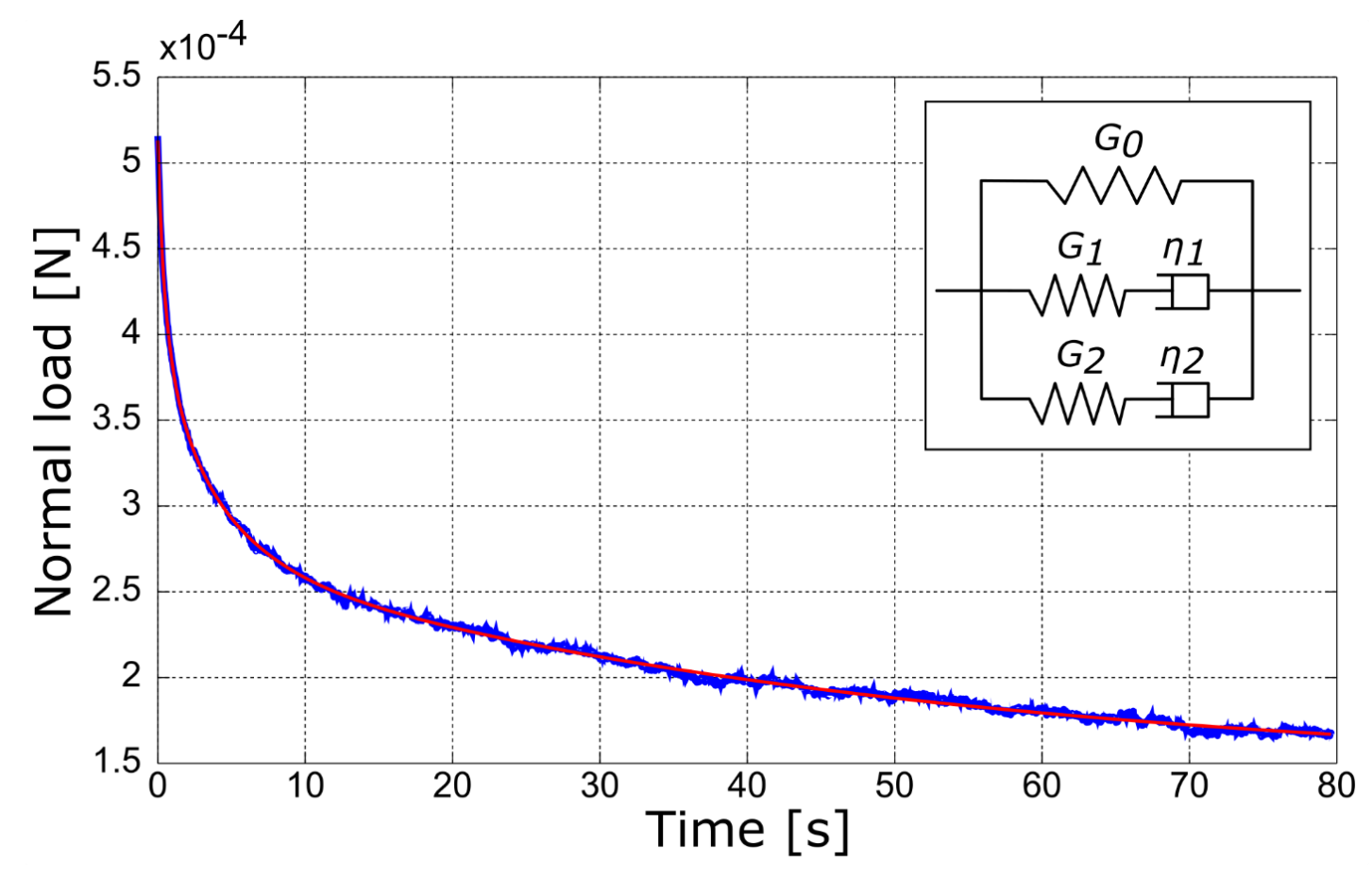

\subsection{Opto-Mechanical Experimentation}

\subsubsection{Device}

The goal of this experiment was to combine mechanical stress with an optical imaging technique to observe the response of the internal fibers to a macroscopic stress. 
Combining the confocal microscope with a micro-tensile device allowed us to visualize the internal network of collagen under stretching. The behavior of the fibers during tensile testing was analyzed using digital image correlation.

The tensile device (DEBEN, 500N Compact Microtest ${ }^{\circledR}$ ) was placed in situ in the confocal microscope. The following tensile parameters were used: a velocity of $2 \mathrm{~mm} / \mathrm{s}$ and a maximum engineering strain of $2.5 \%$.

We captured stacks of images with slice dimensions of $1024 \times 1024$ pixels at a resolution of $0.5 \mu \mathrm{m} / \mathrm{pixel}$ and $1 \mu \mathrm{m}$ between each slice. The top of the dermis was analyzed, which is where collagen is synthesized. Each stack ( $\sim 0 \mu \mu \mathrm{m}$ in Z) was transformed into a single image using maximum-intensity projection (Image ${ }^{\circledR}$ software). This image represents the condition of the area at a specific macroscopic strain. Finally, we obtained two images per sample that displayed internal views of the samples at their initial state and after a strain of $2.5 \%$.

\subsubsection{Digital Image Correlation}

The developed method is based on digital image correlation (DIC $)^{26}$ and was previously described by Molimard et al. ${ }^{17}$ Only the basic principle is given here.

\subsubsection{2D Digital Image Correlation}

Assuming a reference image $\mathrm{im}_{0}$ described by $f(r, s)$, a deformed image $\mathrm{im}_{1}$ of $\mathrm{im}_{0}$ after a small deformation is described by $g(r, s)$ :

$$
g(r, s)=f\left(r-\delta_{x}, s-\delta_{y}\right)+b(r, s)
$$

where $\delta_{x}$ and $\delta_{y}$ are the components of the displacement of $i m_{1}$ and $b(r, s)$ is the noise. One method to determine $\delta_{x}$ and $\delta_{y}$ is to maximize function $h$, which is defined by:

$$
h(r, s)=(g \otimes f)(r, s)=\int_{-\infty}^{+\infty} \int_{-\infty}^{+\infty} g(a, b) f(a-r, b-s) \mathrm{d} a \mathrm{~d} b
$$

where $\otimes$ denotes the cross-correlation product. The position of the maximum value of $h(r, s)$ corresponds to the maximum displacement probability $\left(\delta_{x}, \delta_{y}\right)$.

Equation 14 is used at a local scale on the region of interest (ROI). To provide a final displacement field, the procedure is repeated for regions located all over the map.

\subsubsection{Sub-pixel Algorithm}

The sub-pixel algorithm is based on a phase analysis of each ROI before and after mechanical stimulation. Each phase is proportional to the displacement normal to the corresponding direction. In the absence of a phase jump, the displacements can be related to any defined phase using the following relationship: 


$$
\left\{\begin{array}{c}
\vdots \\
\Delta \phi_{\theta}^{i} \\
\vdots
\end{array}\right\}=\left[\begin{array}{cc}
\vdots & \vdots \\
\frac{2 \pi}{p_{\theta}^{i}} \cos \theta^{i} & \frac{2 \pi}{p_{\theta}^{i}} \sin \theta^{i} \\
\vdots & \vdots \\
= & \vdots
\end{array}\right]\left\{\begin{array}{l}
\delta_{x} \\
\delta_{y}
\end{array}\right\}
$$

where $\left\{\begin{array}{c}\vdots \\ \Delta \phi_{\theta}^{i} \\ \vdots\end{array}\right\}$ is a vector containing all of the phase differences, $\left\{\begin{array}{l}\delta_{x} \\ \delta_{y}\end{array}\right\}$ is the displacement vector, and $A$ is the sensitivity matrix. $A$ is a rectangular matrix with 2 rows and as many lines as defined phases, which can be up to 255 under usual conditions.

The displacements can then be derived from Eq.(15) using the pseudo-inverse of $A$. This operation is possible if $\operatorname{det}\left(\left(A^{t} A\right)\right) \neq 0$. In practice, this indicates that at least two phases along two different directions exist.

$$
\left\{\begin{array}{l}
\delta_{x} \\
\delta_{y}
\end{array}\right\}=\left(\mathrm{A}^{\mathrm{t} A}\right)^{-1} \mathrm{~A}^{\mathrm{t}}\left\{\begin{array}{c}
\vdots \\
\Delta \phi_{\theta}^{i} \\
\vdots
\end{array}\right\}
$$

\subsubsection{Strain Fields}

Finally, the strain fields $\left(\varepsilon_{X X}\right.$ and $\left.\varepsilon_{Y Y}\right)$ were obtained using a 2D local polynomial approach. This method was proven to be 8 times more efficient than classical finite difference methods. ${ }^{1}$

\subsubsection{Global Strain Values and Poisson's Ratio Assessment}

The distribution of strain fields was supposed to follow a normal distribution, and the global strain values were evaluated as the mean. Using those values, we determined the Poisson's ratio, $v$, which is defined for a tensile test as:

$$
v=\frac{-\varepsilon_{Y Y}}{\varepsilon_{X X}}
$$

\subsubsection{Determination of Mechanical Properties at the Microscopic Scale}

Following linear elasticity and small deformation hypotheses, we can determine the mechanical properties at a local level. Here, considering the microscope magnification, the local level is a microscopic one, corresponding to the fiber scale.

Using the global strain values, the Poisson's ratio and the elastic modulus, which was characterized by indentation and corrected with the experimental value of the Poisson's ratio, we determined the stress along $\vec{x}, \sigma_{X X}[\mathrm{~Pa}]$, which is defined for a tensile test as:

$$
\sigma_{X X}=\frac{E}{1-v^{2}}\left(\varepsilon_{X X}+v \varepsilon_{Y Y}\right)
$$


The work of elastic deformation along $\vec{x}, W_{e X}[\mathrm{~J}]$, can be determined using the volume of optical acquisition, $V\left[\mathrm{~m}^{3}\right]$, as follows:

$$
W_{e X}=\frac{1}{2} E \varepsilon_{X X}^{2} V
$$

Finally, using the first theorem of Castigliano (Eq.20), forces along $\vec{x}, F_{X}[\mathrm{~N}]$, that are applied to the fibers in this volume are determined by Eq. (21):

$$
\begin{aligned}
& W_{e X}=\frac{1}{2} F_{X} \delta_{x} \\
& F_{X}=\frac{2 W_{e X}}{\delta_{x}}
\end{aligned}
$$

\subsection{Statistics}

Mean and standard deviation values were calculated for the results of the same tissue sample group. The results are presented as $M \pm S D$ with $M=$ mean and SD = standard deviation. Moreover, unpaired Student's $t$ tests were performed between two groups, and the $p$ values, $p$, are presented.

\section{Results}

\subsection{Micro-structure Observations}

\subsubsection{Acellular Matrix}

The acellular matrix, basis of next samples, is shown in Fig. 8a. It is a collagen-chitosanglycosaminoglycan scaffold which could be visualized in both channels thanks to the second harmonic generation of collagen and the auto-fluorescence of chitosan, previously described in the literature. ${ }^{4}$

\subsubsection{Reconstructed Dermis}

Confocal images of reconstructed dermis samples were acquired (Fig. 4). Because two channels were used, we were able to visualize the signal of the artificial matrix (visible by auto-fluorescence of the chitosan in blue) and the signal of the human collagen (visible by second harmonic generation of the collagen in green). From these images we observed that the fibroblasts induced the neo-synthesis of collagen. Moreover, the cells from the younger man synthesized more collagen. Indeed, the density of synthetized collagen (in green) is equivalent at the top of the sample but decrease strongly in depth with the use of the older man's cells. 
Figure 4: ZX view of reconstructed dermis samples obtained using bi-photonic confocal microscopy and plots of the density of signals in each image through depth.
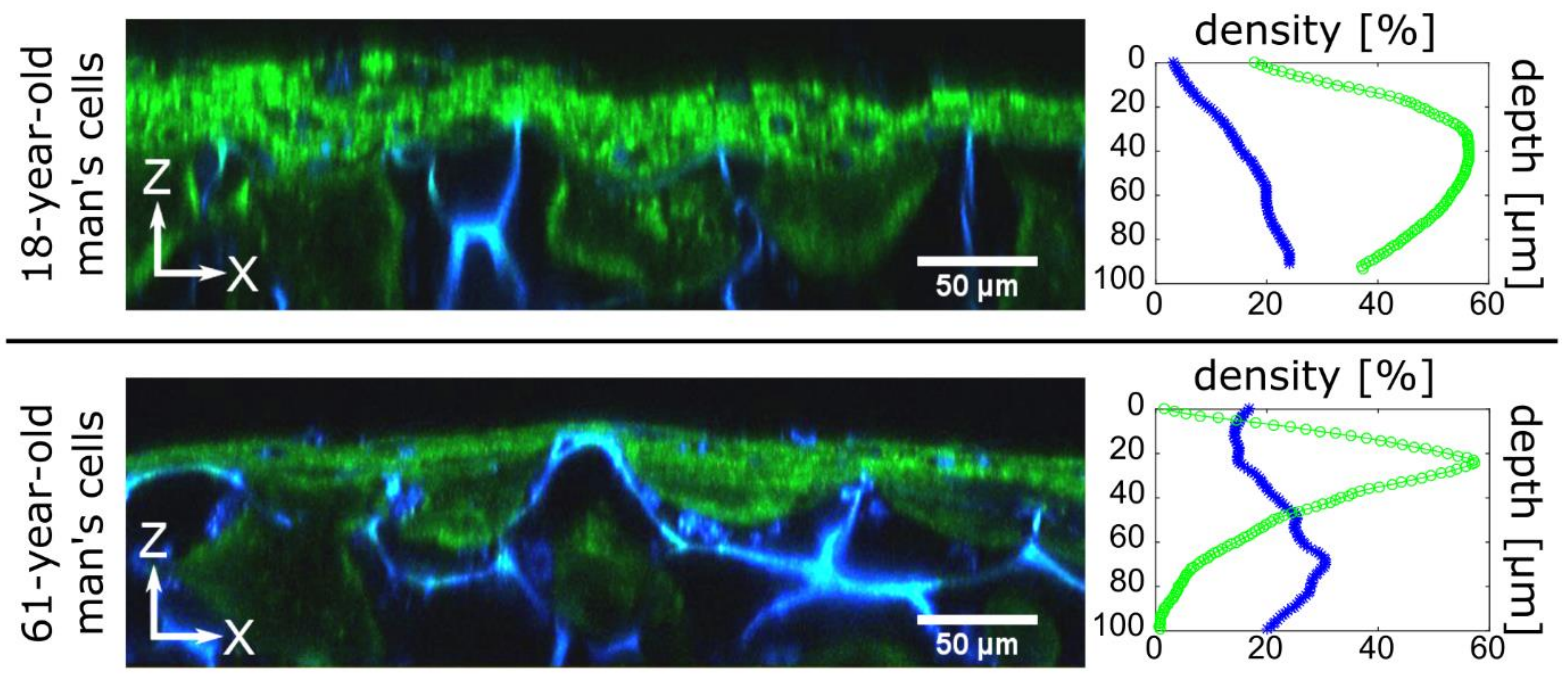

\subsubsection{Reconstructed Skin}

Confocal images were acquired for the reconstructed skin sample (Fig. 5). At the bottom of the images, we can observe the matrix (visible by auto-fluorescence of the chitosan in blue) and collagen neo-synthesis (visible by second harmonic generation in green). Keratinocytes were seeded on top of this layer. As described in the literature, these cells increase the production of keratin during their differentiation. ${ }^{28}$ This produced a highly fluorescent region that corresponded with the keratinizing layer (stratum corneum) on the surface of the sample. Keratinocytes in the lower section of this reconstructed epidermis produced less keratin; they are not visible in these images because their fluorescent intensity is significantly lower than that of the stratum corneum. The cells from the younger man synthesized more collagen. Indeed, the density of synthetized collagen (in green) is lower with the use of the older man's cells (30 and 18\%, with the use of the younger man's cells and of the older man's cells, respectively). 
Figure 5: ZX view of reconstructed skin samples obtained using bi-photonic confocal microscopy and plots of the density of signals in each image through depth.
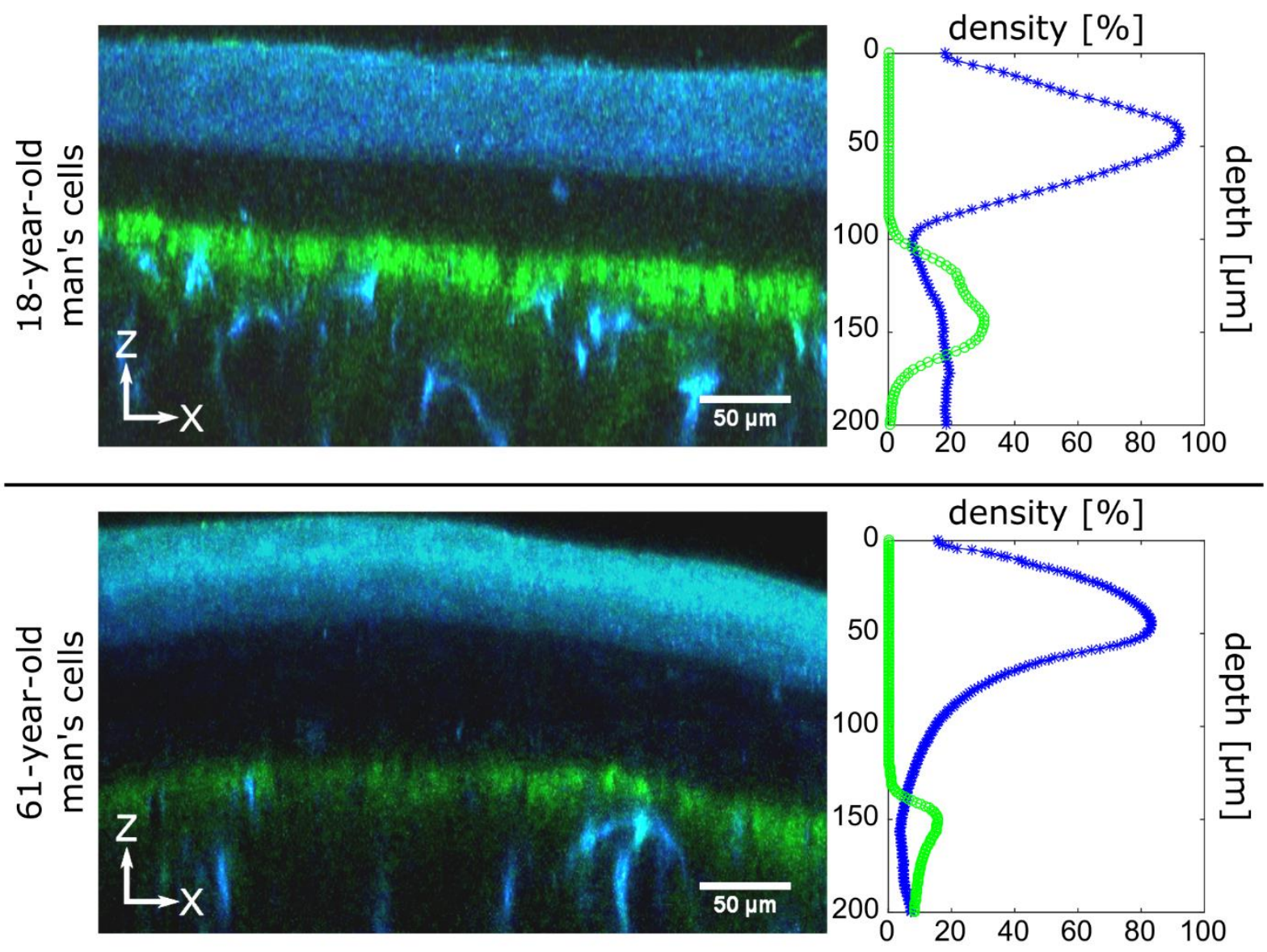

\subsection{Mechanical Results}

\subsubsection{Indentation}

Reduced elastic modulus obtained from the indentation tests are presented in Fig. 6.

The results reveal increasing values $(p<0.001)$ from the acellular matrix $(14.8 \pm 1.1 \mathrm{kPa})$ to the reconstructed dermis $(59.1 \pm 6.6 \mathrm{kPa})$ and finally the reconstructed skin $(160.3 \pm$ $32.5 \mathrm{kPa}$ ). We were able to follow the effect of adding cells during the culture of artificial skin. Moreover, the use of cells from the older donor yielded significantly lower values of the reduced elastic modulus $(-13.1 \mathrm{kPa}, p<0.01$, and $-76.6 \mathrm{kPa}, p<0.001$, for the dermis and skin, respectively). 
Figure 6: Reduced elastic modulus, $E^{*}$, obtained by indentation test on the tissue engineered samples. $(* p<0.05)$

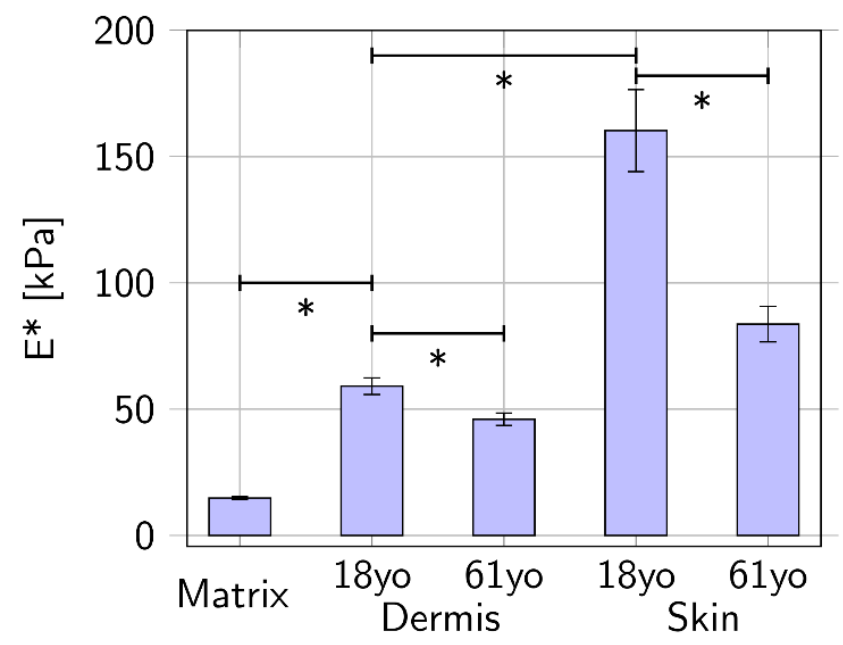

\subsubsection{Relaxation}

The mechanical parameters obtained from the relaxation tests are presented in Fig. 7.

The results exhibit a significant increase $(p<0.001)$ in the instantaneous elastic modulus from the acellular matrix $(3.8 \pm 0.3 \mathrm{kPa})$ to the reconstructed dermis $(8.9 \pm 1.3 \mathrm{kPa})$ and the reconstructed skin $(83.3 \pm 33.1 \mathrm{kPa})$. We are able to monitor the effect of adding cells while culturing the artificial skin. Moreover, the use of cells from the older donor yielded a lower instantaneous elastic modulus $(-5.0 \mathrm{kPa}, p<0.001$, and $-33.3 \mathrm{kPa}, p<0.01$, for the dermis and skin, respectively).

In terms of viscosity, $\eta_{\text {equ }}$ follows the same trend as the instantaneous elastic modulus. The viscosity increased from $2.5 \pm 1.0 \mathrm{kPa}$.s to $7.1 \pm 2.3 \mathrm{kPa}$.s and finally to $150.8 \pm 97.5$ $\mathrm{kPa}$.s for the acellular matrix, reconstructed dermis, and reconstructed skin, respectively. A decrease in the equivalent viscosity was observed for the samples using older male cells (-2.5 kPa.s, $p<0.01$, and $-67.9 \mathrm{kPa} . \mathrm{s}, p<0.05$, for the dermis and skin, respectively). 
Figure 7: Instantaneous elastic modulus, $E_{\text {ins, }}$ and equivalent viscosity, $\eta_{\text {equ, }}$ obtained by relaxation test on the tissue engineered samples. $(* p<0.05)$
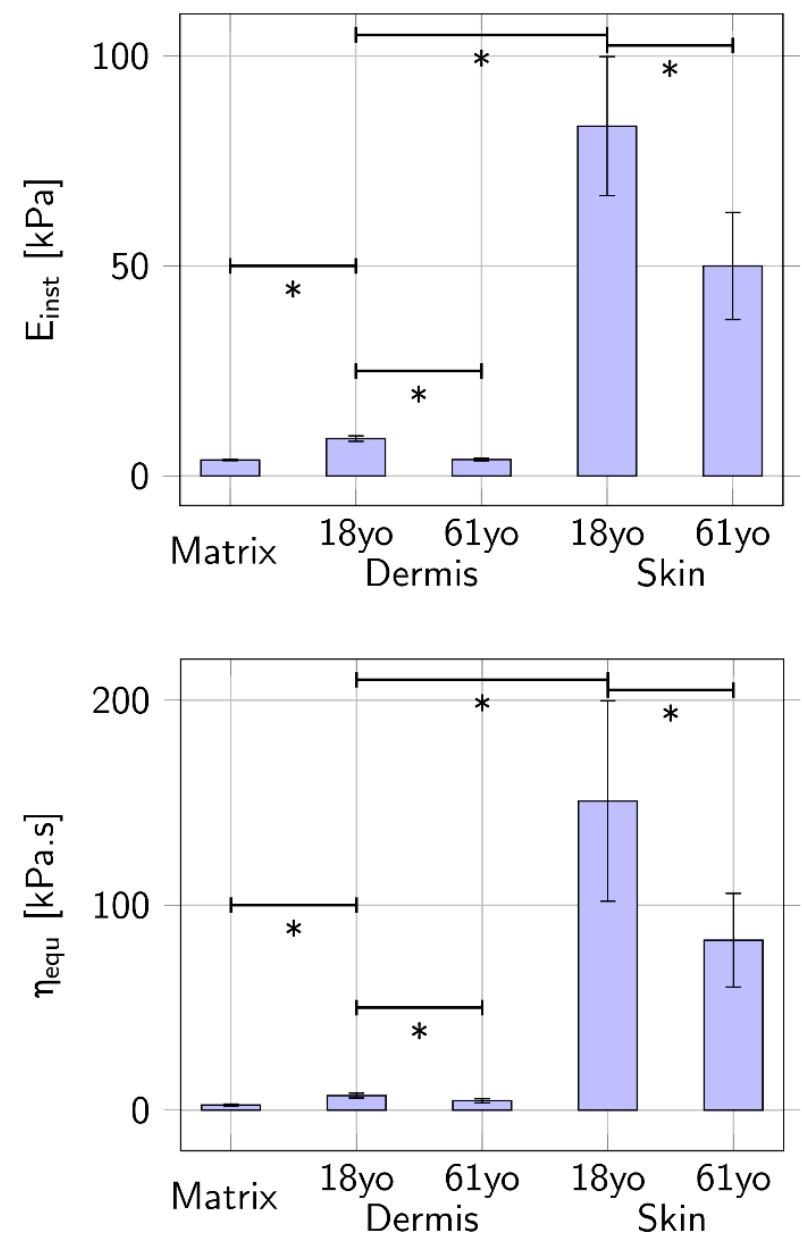

\subsection{Opto-Mechanical Results}

\subsubsection{Displacement Fields and Poisson's Ratio}

For each sample, digital image correlation was applied to the top part of the matrix. An example of this image correlation process on the artificial matrix can be followed in Fig. 8.

Displacement fields (Fig. 8c and 8d) are typical results for a tensile test. After derivation, strain maps (Fig. 8e and 8f) were assessed and the global strains were estimated. Global strains are estimated by fitting the distribution of strain maps with a normal law. Using these parameters, the Poisson's ratio could be experimentally assessed (Table 1). The results exhibit a decrease in the Poisson's ratio from the acellular matrix $(0.42)$ to the reconstructed dermis $(0.21)$ and the reconstructed skin (0.20).

Moreover, the use of cells from the older donor did not yield a significant difference. 
Figure 8: Digital image correlation applied to an acellular matrix sample: biphotonic confocal images (initial state (a) and after a strain of $2.5 \%$ (b)), displacement fields along $X(c)$ and $Y(d)$ evaluated using digital image correlation and strain fields $\varepsilon_{X X}(\mathrm{e})$ and $\varepsilon_{Y Y}(\mathrm{f})$ after derivation.
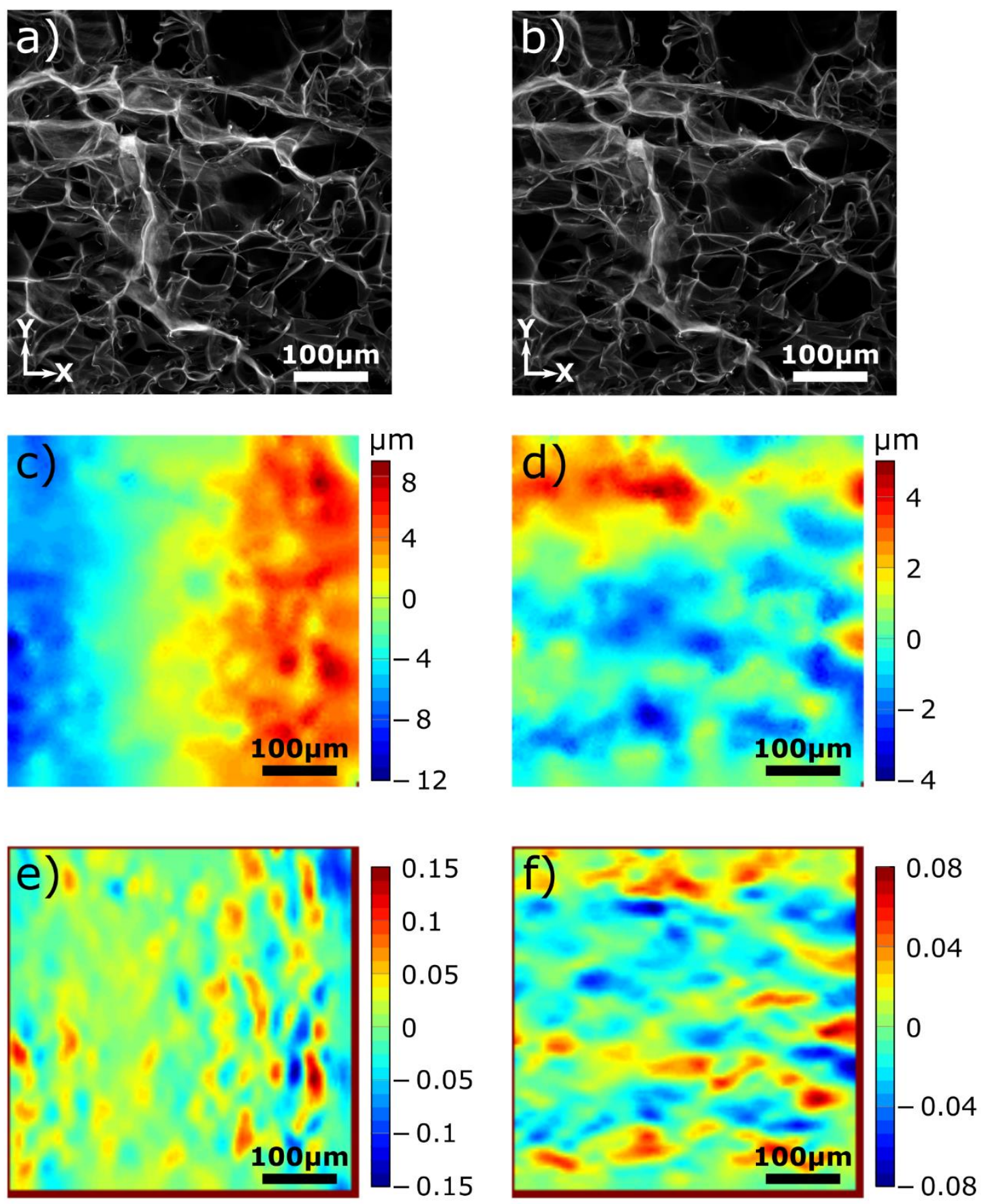
Table 1: Global strains, $\varepsilon x x$ and $\varepsilon y Y$, and Poisson's coefficient, $v$, estimated for each tissue engineered sample.

\begin{tabular}{|c|c|c|c|}
\hline Sample & $\varepsilon_{X X}$ & $\varepsilon_{Y Y}$ & $v$ \\
\hline Acellular matrix & 0.016 & -0.006 & 0.42 \\
\hline Dermis 18yo & 0.016 & -0.003 & 0.21 \\
Dermis 61yo & 0.012 & -0.003 & 0.25 \\
\hline Skin 18yo & 0.014 & -0.003 & 0.20 \\
Skin 61yo & 0.012 & -0.002 & 0.20 \\
\hline
\end{tabular}

\subsubsection{Mechanical Properties at the Microscopic Scale}

Using previous parameters (global strains, Poisson's ratio and elastic modulus estimated by indentation), mechanical properties were calculated at the microscopic scale (Table. 2).

The results exhibit an increase in the internal stress and force from the acellular matrix (239.0 $\mathrm{Pa}$ and $11.6 \mu \mathrm{N}$, respectively) to the reconstructed dermis (948.6 $\mathrm{Pa}$ and $52.9 \mu \mathrm{N}$, respectively) and the reconstructed skin (2278 $\mathrm{Pa}$ and $130.4 \mu \mathrm{N}$, respectively). We are able to monitor the effect of adding cells while culturing the artificial skin.

Moreover, the use of cells from the older donor yielded lower parameters (-389.9 $\mathrm{Pa}$ and $-25.6 \mu \mathrm{N}$ for the reconstructed dermis and $-1238 \mathrm{~Pa}$ and $-74.3 \mu \mathrm{N}$ for the reconstructed skin, respectively). 
Table 2: Mechanical parameters at microscopic level for each tissue engineered sample: stress, $\sigma_{X}$, elastic work, $W_{e X}$, and force, $F_{X}$.

\begin{tabular}{|c|c|c|c|}
\hline Sample & $\sigma_{X}[\mathrm{~Pa}]$ & $W_{e X}[\mathrm{~nJ}]$ & $F_{X}[\mu \mathrm{N}]$ \\
\hline Acellular matrix & 239.0 & 0.312 & 11.6 \\
\hline Dermis 18yo & 948.6 & 1.20 & 52.9 \\
Dermis 61yo & 558.7 & 0.534 & 27.3 \\
\hline Skin 18yo & 2278 & 2.61 & 130.4 \\
Skin 61yo & 1040 & 1.02 & 56.1 \\
\hline
\end{tabular}

\section{Discussion}

\subsection{Structural and Aging Effects}

Our work allowed us to study the effect of aging on artificial skin samples using cells from two donors of different ages (18- and 63-year-old). Mechanical experiments provided information on the elasticity and viscosity of those samples. Using a collagen-chitosanglycosaminoglycan matrix, we can create a reconstructed dermis using fibroblasts. This scaffold is composed of large fibers and is porous enough to be seeded with fibroblasts. After a period of culture, the cells colonized and began to remodel the matrix. The cells degraded the matrix and stimulated neo-synthesis of human collagen, as observed with bi-photonic confocal microscopy. With regards to the mechanical properties, the elastic modulus increased significantly between the matrix and the reconstructed dermis. This result was confirmed by two mechanical tests (indentation \& relaxation). The use of older cells yielded a smaller elastic modulus that was equivalent to the initial matrix. This result is attributed to the less efficient collagen synthesis that was observed for older cells (Fig. $4 b)$.

To reconstruct a layer of skin, keratinocytes were seeded on top of the reconstructed dermis. As observed with confocal microscopy, these cells formed a layer on top of the sample, which is the result of stratification and the complete differentiation of keratinocytes. The skin sample is composed of a rigid layer on top of a fibrillar milieu.

With regards to mechanical properties, the elastic modulus and viscosity increased significantly with the addition of cells. The rigid layer of differentiated keratinocytes maintained the entire structure of the sample, which increased the viscosity. Confocal images revealed similar layers of keratinocytes for matrices seeded with either young or 
old cells. Moreover, seeding keratinocytes on top of the reconstructed dermis stimulated fibroblast metabolism: the synthesis of collagen seemed to be equivalent for young and old cells (Fig. 5a and 5b).

However, under the epidermis, the layer of fibroblasts was larger for matrices seeded with cells from the older donor. This structural configuration had a lower elastic modulus, as shown in Fig. 7.

\subsection{Opto-mechanical Assessments}

We know that in vivo skin aging induces a loss of collagen and elastic fiber density that decreases internal tension, as revealed by Pailler-Mattei et al. ${ }^{19}$ The goal of our optomechanical experiments was to reproduce this internal tension using in vitro samples. The microstructure of the sample, before and after a macroscopic strain of $2.5 \%$, was analyzed using digital image correlation to assess the displacement and strain fields (after 2D local polynomial derivation). It leads to the evaluation of the Poisson's ratio and the internal stress and force in the sample. This internal force reflects the ability of the sample to resist deformation.

The Poisson's ratio could be experimentally assessed (Table 1). The acellular matrix exhibits a value of 0.42 which is not far away from the usually used value for an incompressible material $(0.45-0.5)$. The addition of cells in the acellular matrix induces a strong decrease of this parameter to a value of 0.2 . This value tends to the experimental values assessed in vivo by Boyer et al. ${ }^{3}$

Internal stress and force increased with the addition of cells during the manufacturing of the reconstructed skin (Table 2). This result emphasizes the participation of fibroblastsynthesized collagen during dermis culture and the addition of the stratified epidermis, which is composed of differentiated keratinocytes, in the manufacturing of reconstructed skin. Moreover, the results revealed a decrease in the internal stresses and forces when cells from the older male donor were used. This is attributed to the less efficient synthesis of collagen, which was visualized using bi-photonic confocal microscopy.

\section{Conclusion}

Measuring the visco-elastic properties and the Poisson's ratio of artificial skin samples are key initiatives to better understand their mechanical behavior.

Complete device and methods for the in vitro assessment of those properties at macroscopic scale were presented (indentation and relaxation tests). The results were obtained for artificial skin samples developed by BASF ${ }^{\circledR}$ Beauty Care Solutions France; a variety of skin models were selected, based on manufacturing steps, and analyzed. The results clearly demonstrate the effect of those steps on the mechanical properties of the skin substitutes at macroscopic level. Moreover, the effect of aging was studied using senescence models (cells from the older male donor). 
Confocal microscopy images were acquired to better understand the structural variations between samples. The images clearly show the effect of the cell type on collagen synthesis. Indeed, cells from the older man were less efficient at synthesizing collagen, which explains the decreased mechanical properties.

Finally, opto-mechanical experiments were performed, which combined bi-photonic confocal microscopy with a tensile device. The micro-structure, before and after $2.5 \%$ strain, was compared using digital image correlation. The displacement and strains that were obtained were used to estimate the Poisson's ratio of the samples. The values of this parameter for our skin equivalent samples vary between 0.2 and 0.25 . Also, mechanical properties at the microscopic scale were evaluated. This experiment demonstrates how much a sample can resist a specific strain. Senescence models are less efficient than normal ones, which can be correlated to in vivo observations for the loss of tension in the skin that is observed with aging.

\section{Acknowledgements}

We would like to convey our deepest gratitude to $\mathrm{BASF}^{\circledR}$ Beauty Care Solutions France (F-69007 Lyon) for providing us with the in vitro samples.

We would also like to thank the IVTV (ANR-10-EQPX-06-01) team for their help during imaging.

\subsection{Conflict of interest}

No conflicts of interest present in this study. 


\section{References}

1. Berge-Gras, R. and J. Molimard. Optimisation de la dérivation en fonction de la résolution et de la résolution spatiale sur les déformations. In: XIXème Congrès Français de Mécanique, AFM, Marseille, France, 2009.

2. Boyer, G., L. Laquieze, A. Le Bot, S. Laquieze and H. Zahouani. Dynamic indentation on human skin in vivo: ageing effects. Skin Res. Technol. 15:55-67, 2009.

3. Boyer, G., J. Molimard, M. Ben Tkaya, H. Zahouani, M. Pericoi and S. Avril. Assessment of the in-plane biomechanical properties of human skin using a finite element model updating approach combined with an optical full-field measurement on a new tensile device. J. Mech. Behav. Biomed. Mater. 27:273-82, 2013.

4. Chen, W.-L., C.-H. Huang, L.-L. Chiou, T.-H. Chen, Y.-Y. Huang, C.-C. Jiang and H.$\mathrm{S}$. Lee. Multiphoton imaging and quantitative analysis of collagen production by chondrogenic human mesenchymal stem cells cultured in chitosan scaffold. Tissue Eng. Part C Methods 16:913-920, 2010.

5. Cheung, H.Y., K.T. Lau, T.P. Lu and D. Hui. A critical review on polymer-based bioengineered materials for scaffold development. Compos. Part B Eng. 38:291-300, 2007.

6. Drury, J.L. and D.J. Mooney. Hydrogels for tissue engineering: scaffold design variables and applications. Biomaterials 24:4337-4351, 2003.

7. Duplan-Perrat, F., O. Damour, C. Montrocher, S. Peyrol, G. Grenier, M.P. Jacob and $F$. Braye. Keratinocytes influence the maturation and organization of the elastin network in a skin equivalent. J. Invest. Dermatol. 114:365-370, 2000.

8. Ebersole, G.C., P.M. Anderson and H.M. Powell. Epidermal differentiation governs engineered skin biomechanics. J. Biomech. 43:3183-3190, 2010.

9. Echinard, C. Under the epidermis, the dermis, or how to interpret skin cultures? Ann. Chir. Plast. Esthet. 43:197-205, 1998.

10. Gerhardt, L.-C., J. Schmidt, J.A. Sanz-Herrera, F.P.T. Baaijens, T. Ansari, G.W.M. Peters and C.W.J. Oomens. A novel method for visualising and quantifying through-plane skin layer deformations. J. Mech. Behav. Biomed. Mater. 14:199-207, 2012.

11. Hidaka, K., L. Moine, G. Collin, D. Labarre, J. Louis Grossiord, N. Huang, K. Osuga, $\mathrm{S}$. Wada and A. Laurent. Elasticity and viscoelasticity of embolization microspheres. J. Mech. Behav. Biomed. Mater. 4:2161-2167, 2011.

12. Jachowicz, J., R. Mcmullen and D. Prettypaul. Indentometric analysis of in vivo skin and comparison with artificial skin models. Skin Res. Technol. 13:299-309, 2007.

13. Johnson, K.L., K. Kendall, and A.D. Roberts. Surface energy and the contact of elastic solids. Proc. R. Soc. Lond. A 324(1558):301-313, 1971. 
14. Kanungo, B.P. and L.J. Gibson. Density-property relationships in collagenglycosaminoglycan scaffolds. Acta Biomater. 6:344-353, 2010.

15. Lamers, E., T.H.S. Van Kempen, F.P.T. Baaijens, G.W.M. Peters and C.W.J. Oomens. Large amplitude oscillatory shear properties of human skin. J. Mech. Behav. Biomed. Mater. 28:462-70, 2013.

16. Mano, J.F., G.A. Silva, H.S. Azevedo, P.B. Malafaya, R.A. Sousa, S.S. Silva, L.F. Boesel, J.M. Oliveira, T.C. Santos, A.P. Marques, N.M. Neves and R.L. Reis. Natural origin biodegradable systems in tissue engineering and regenerative medicine: present status and some moving trends. J. R. Soc. Interface 4:999-1030, 2007.

17. Molimard, J., G. Boyer and H. Zahouani. Frequency-based image analysis of random patterns: an alternative way to classical stereocorrelation. J. Korean Soc. Nondestruc. Test. 30:181-193, 2010.

18. Nelder, J.A. and R. Mead. A simplex algorithm for function minimization. Comput. J. 7:308-313, 1965.

19. Pailler-Mattei, C., R. Debret, R. Vargiolu, P. Sommer and H. Zahouani. In vivo skin biophysical behaviour and surface topography as a function of ageing. J. Mech. Behav. Biomed. Mater. 28:474-83, 2013.

20. Pailler-Mattei, C., L. Laquièze, R. Debret, S. Tupin, G. Aimond, P. Sommer and H. Zahouani. Rheological behaviour of reconstructed skin. J. Mech. Behav. Biomed. Mater. 37C:251-263, 2014.

21. Peppas, N.A., J.Z. Hilt, A. Khademhosseini and R. Langer. Hydrogels in biology and medicine: from molecular principles to bionanotechnology. Adv. Mater. 18:1345-1360, 2006.

22. Roeder, B.A., K. Kokini, J.E. Sturgis, J.P. Robinson and S.L. Voytik-Harbin. Tensile mechanical properties of three-dimensional type I collagen extracellular matrices with varied microstructure. J. Biomech. Eng. 124(2):214, 2002.

23. Shahabeddin, L., F. Berthod, O. Damour and C. Collombel. Characterization of skin reconstructed on a chitosan-cross-linked collagen-glycosaminoglycan matrix. Skin Pharmacol. 3:107-114, 1990.

24. Silver, F.H., J.W. Freeman and D. DeVore. Viscoelastic properties of human skin and processed dermis. Skin Res. Technol. 7:18-23, 2001.

25. Silver, F.H., L.M. Siperko and G.P. Seehra. Mechanobiology of force transduction in dermal tissue. Skin Res. Tech. 9:3-23, 2003.

26. Sutton, M.A., J.-J. Orteu and H. Schreier. Image Correlation for Shape, Motion and Deformation Measurements-Basic Concepts, Theory and Applications. New York: Springer-Verlag, 2009, $321 \mathrm{pp}$.

27. Voytik-Harbin, S.L., B.A. Roeder, J.E. Sturgis, K. Kokini and J.P. Robinson. Simultaneous mechanical loading and confocal reflection microscopy for three- 
dimensional microbiomechanical analysis of biomaterials and tissue constructs. Microsc. Microanal. 9:74-85, 2003.

28. Wu, Y., P. Xi, J.Y. Qu, T.-H. Cheung and M.-Y. Yu. Depth-resolved fluorescence spectroscopy reveals layered structure of tissue. Opt. Express. 12(14):3218, 2004.

29. Zahouani, H., C. Pailler-Mattei, B. Sohm, R. Vargiolu, V. Cenizo and R. Debret. Characterization of the mechanical properties of a dermal equivalent compared with human skin in vivo by indentation and static friction tests. Skin Res. Technol. 15(1):6876, 2009. 